\title{
XV. \\ Ueber das galvanische Verhalten und die Leitungsfähigkeit der Mineralkörper als Kennzeichen.
}

\author{
Von \\ Prof. $\boldsymbol{v}$. Kobelt.
}

(Gel. Anzeigen der $k$. baier. Akademic der Wissenschaften.)

Bekanntlich werden zur Erzeugung eines galvanischen Stromes drei chemisch verschiedene leitende Substanzen erfordert, wovon die cine gervöhnlich ein zusammengesetztes Fluidum ist. Wenn der galvanische Strom eine hinlängliche Stärke besitzt, so wird der flüssige Leiter zersetzt und besleltt er aus einer geeigneten Salzaullósung, so begiebt sich die Säure oder ilır Stellvertreter zu dem positiven festen Leiter oder zur Anode, die Base dagegen zu dem negativen oder zur Kalhode. Bei aufgelissten Metallsalzen wird das Mrtall auf der Kathode oft regulinisch ausgeschieden. Es schien mir zur Erweiterung der mineralogischen Kennzeichen von Interesse, das galvanische Verhalten vorzüglich der natürlich vorkommenden metallischen Verbindungen zu untersuchen, zunäclist in Combination mit Zink, gregen welches sie sämmtlich negativ sind und als Kathoden gebraucht wurden. lla es mir dabei nur un leicht nachweisbare Kennzeichen zu thun war, so wahlte ich folgendes Verfahren. Ich verschalfte mir mehrere liluppen von Zinkblech, indem ich solches Blech zu Streifen von ohngefälır 6 Zoll Länğge und $\frac{1}{4}$ Zoll breit schnitt und die Enden zusammeuhog. Das zu prüfende Mineral wurde als ein Stück von einigen linien Grösse mit den Enden der Kluppe gefisst und in eine Auflösung von Kupfervitriol eingesenkt. Der dabei entstehende galvanische Strom, wenn das Mineral pin Leiter, ist schnell vorübergehend, da sich das Zink sogleich mit Kupfer belegt und die weitere galranische Wirkung auf die Probe selbst nur von selır geringen Einflusse ist; gleichwohl werden dabei alle besseren Leiter leicht erkannt, indem sie sich melı oder weniger schnell mit Kupfer belegen. Da nur einige Zinkstreifen und eine kleine Schaale mit Kupfervitriollösung zu den Versuchen nothwendig, so dürften die daraus 
sich ergebenden Kennzeichen als Controle oder Zugabe anderer einigen Werth haben. Bei Anstellung der Versuche selbst ist nur zu hemerken, dass die Enden der Kluppe rein gefeilt und die Proben ganz frisch geschlagene Bruch- oder Spaltungsstüclie sein mussen und dass man beim Einklemmen oder Fassen mit der Kluppe daranf achte, das Zink wo möglich mit Flächen der Probe, nicht mit Ecken oder scharfen Kanten in Beruilurung zu bringen.

Die Versuche wurden bei gewöhnlicher Temperatur angestellt, bei erhöhter wird die Wirkung verstärkt. Das Eintauchen der Proben dlauerte 1 Minute, dann wurden sie in Wasser getaucht und abgespült und mit einem Tuche getrocknet.

Es zeigten sich als gute Leiter und belegten sich schnell mit Kupfer:

Amalgam.

Antimon gediegen.

Anlimonsilber.

Antimonfahlerz.

Arsenik gediegen.

Arsenikfahlerz.

Arsenikkies prismatischirr.

Arsenikkies axotomer.

Blältererz.

Bleiglanz.

Burıkupfererz.

Glanzkobalı.

Gold gediegen.

Graphit.

Kupferglanz.

Kupferkies.

Magneteisenel:z.

Magnetkies.

Nickel wismuthglanz.

Palladium gediegen.

Platin gediegen.

Rotbnickelkies.

Sclrifttellur.

Schwefelkies tess.

Scbwefelkies rhomb. 
Scliwefelkobalt.

Selenblei.

Silber gediegen.

Sprisskobalt.

Tesseralkies von Mollun $\mathrm{Fe}_{\mathrm{A}} \mathrm{As}_{3}$.

Weisskupfererz.

Wismuth gediegen.

Weun man ein Stückchen liupferkies nur an den Berührungspunkten mit Kupfer überiaulen lässt und danu hernusuimmt und abtrocknet, so überläuft es in kurzer Zeit mil schönen bunten Farben. Ich halie schon früher einmal diese interessante Erscheinung erwähnt, die man bei grösseren Stücken hervorbringen kann, wenn man sic mit frischen Bruchflächen auf ein Zinkblech legt und so einige Secunden lang in Kupfervitriol eintaucht. Eisenkies zeigt die Erscheinung nicht, Nagnetkies aber zeigt blaue Anlauffarbe. -

Weniger gut leitend als die vorigen belegten sich in der Nähe der Berūhrungsstellen mit Kupfer:

Antimonsilberblende.

Arsensilberblende.

Bournonit (schwach).

Glaserz.

Ilmenit (schwach).

Molybdänglanz.

Tellur gediegen.

Titaneisen von Egersund (schwach).

Wismuthglanz (schwach).

Zinkblende, wenn eine Spaltungsfläche mit dem Zink berührt wurde.

Zinnstein in den schwärzlichbraunen Krystallen. IIier zeigte sich die seltsame Erscheinung, dass von einem und demselben Krystalle die innern gelblichen stark durchscheinenden Theile fast gar nicht mit Kupfer belegt wurden, während sich die äusseren sclıwärzlichen und fast undurchsichtigen gut leitend zeigten. Es kounte an letztern keine sichtbare Einmengung bomerkt werden, auch keine Wirkung auf die Magnetnadel.

Nicht leitend zeigten sich unter diesen Verhältnissen:

Antimonblende.

Antimonglanz. 
Braunit.

Chlorquecksilber.

Chlorsilber.

Chromeisenerz.

Dulrenoysit.

Eisenglanz vom Fichtelgebiro.

Eisenglanz von Elba.

Eisenglanz fasriger.

Federerz.

Franklinit.

Geokronit.

Hauerit.

Hausmannit.

Jamesonit.

Kibdelophan (Spur).

Manganglanz.

Manganit.

Operment.

Psilomelan.

Pyrolusit.

Realgar.

Rothkupfererz.

Rutil.

Silberfahlerz von Freiberg.

Sprödglaserz (Spur).

Tantalit.

Uranpecherz.

Wolfram.

Yttertantal.

Zinkenit.

Zinnober.

Zinnerz kornisches.

Es sind auf diese Weise mehrere ähnliche Mineralien sehr leicht zu unterscheiden, z. B. Magneteisenerz von Titaneisen, Chromeisenerz etc., Bleiglanz von den verschiedenen Verbindungen von Schwefelblei und Schwefelantimon, Fahlerz von Dufrenoysit, Bournonit etc., Graphit von Molybdänglanz, manchem grauen Glimmerschiefer etc.

Die meisten Oxydverhindungen der Metalle zeigen, wie auch 
die nichtmetallischen Substanzen, keine zur Stromerzeugung hin!ängliche Leitungsfähigkeit, wenn sie auch nicht zu ausgemachten Isolatoren gehören; manche Metallverbindungen werden aber leitend, wenn sie vorher vor dem Löthrühre im Reduktionsfener geschmolzen wurlen, wie $z$. B. die Kupferoxydverbindungen, Chlorkupler, Chlorsilber etc. Wenn Rotheisenerz und Brauncisenerz im Reduktionsfeuer anhaltend scharf geglüht werden, ülerlaufen sie ebenfalls mit Kupfer; beim Pyrolusit und Manganit zeigt sich nach dem Glühen zwar kein deutliches Ueberlaufen mit kupfer, doch wird etwas davon abgesetzt, wie sich nach dem Befeuchten mit Salzsäure vor dem Lülhrobre zeigen lässt. Das Verhalten der Manganerze überhaupt ist auffallend, da wenigstens das Manganperoxyd gewölinlich zu den besseru Leitern gerechnet wird. -

Interessant ist auch das Verhalten der Anthracite. Ich untersuchte mehrere von verschiedenen Fundorten. Als gut leitend zeigte sich ein Anthracit von lichter, stahlgrauer Farbe, angeblich von Schönfeld im Erzgebirge; er ist schiefrig und von gelblichem Kalksinter durchsetzt. Eine andere Varietăt von Lischwitz bei Gera und von Quetschen bei Hof, mit graulichem Kalkspath verwachsen auf dichtem Uebergangskalk, belegte sich wälırend einer Minute*) ebenfalls, wenigstens um die Berührungsstellen. Die meisten Anthracite aber, die ich prüfen konnte, zeigten kein Belegen, so die Anthracite von Hattingen im Jülch'schen, von Südwallis, auch ein schwärzlichgrauer von Schönfeld und die ausgezeichneten Varietäten von Schnilkill in Nordamerika.

Es werden zah!reiche Beispiele angeführt, wie durchsetzende Basalte, Dolerite, Phonolithe, Porphyre und Melaphyre, sogar Diorite, Stein- und Braunkollen in ihrer unmittelbaren Nähe in Anthracit verwandelt haben und die Geologen heben diesen Umstand besonders hervor, um damit die pyrogene Natur dieser

*) Das Eintauchen auf die Dauer einer Minute ist zweckmässig, denn bei längeren Eintauchen wirkt die leitende Substanz nicht mehr allein zur Belegung, sondern das einmal angeschossene Kupfer wird nun der Leiter und das langsame Ueberziehen der Masse erfolgt, wie das Ueberziehen der Farben in der Galvanographie, mehr durch Ausbreitung und Ueberwuchern der Kupfertheilchen, die an ein bereits vorhandenes sich anschliessen, als durch die Leitung der Unterlage. 
Gesteine darzuthun. Von besonderem Interesse ist das Vorkommen eines solchen Falles am Meissner in Hessen, wo einr Basaltmasse von mehreren 100 Fuss Mächligkeit, ein Braunkohlenlager, von dem sie nur durch eine dünne Thonschicht getrennt ist, überdeclit. Die Braunkohle ist abwärts, auf $7-8$ Fuss weit, mehr oder weniger verändert, und die dem Basalt zunächst liegenden Theile zeigen eine stängliche Absonderung und sind in Anthracit verwandelt, wälrend sich weiter Uebergïnge bis zur unveränderten Bratnkohle verfolgen lassen. Ich habe diese Stangenkohle rom Mleissner geprüft, sie zeigte aber durchaus kein Belergen. Yor dem Löthrohre verhielt sie sich übrigens wie die meisten Anthracite, indem sic in Kolben nur Wasser und keinen Theer absetzte, jedoch einen mehr brenzlichen Geruch entwickelte als es bei anderen der Fall ist. Um diese Erscheinungen aufzuklären, stellte jch einige Versuche mit Schwarzkohlen an. lch erhitzte kleine Stücke in einem lose bedeckten Platintiegel über der Weingeistlampe, bis sich keine brennbaren Gasarten mehr entwickelten. Dabei waren die Kohlen durch ihre ganze Masse in's Glühen gekommen. Ich untersuchte nun solche leicht gebrannte Koks, sie zeigten sich aber eben so wenig leitend als die Steinkohlen selbst, nur an einzelnen Stellen in der Nähe des Zinks erschien an einigen ein Kupferanflug. Wenn ich aber diese Koks weiter in der Pincette ror dem Löhrohre durchglühte, so zeigten sie sich nun gut leitend und überliefen schnell mit Kupfer.

So verhalten sich alle Stein- und Braunkolilen, und auch die gewöhnlichen Holzkohlen werden erst leitend, wenn sie in einem Hitzgrade, wie ihn das Löthrohr hervorbringt, durchgeglüht werden. Es geht daraus deutlich herror, tass die Stangonkohle des Meissner keiner hefligen Gluhhilse ausgesetot gewesen sein kann, denn Koks, die einmal so stark gebramnt wurden, dass sie gute Leiter sind, verlieren diese Eigenschaft nicht, auch wenn sie feuchter Luft ausgesetzt sind und Wasser aufgenommen haben, wie überhaupt diese Körper ausser ilhrer Verbrennlichkeit zu den unveränderlichsten gehüren. - Nun soltte man freilich denken, dass eine feuerflüssige Basaltmasse von solcher Mächtigkeit, wie die am Meissner, besonders als Ueberlagerungsmasse eine bedeutende Hitze auf die Unterlage ausgeübt habe, und es bleibt das angegebene Verhalten der 
Stangenkohle immer räthselhaft, wenn man auch amimmt, lass die Zwischenlage des Thons (ist dieser hartgebrannt?) die Wirkung auf die Kohlen geschwächt habe. Leider fehlt es mir an Vorkommnissen voll Anthracit, welcher aus Kohlen durch unmiltelbare Berübrung mit vulkanischen oder jyrogenen Gesteinen gebildet wurde. Den Geologen wird aber das hier erwihnte Experiment zur Prüfung der galvanischen Leitungsfahiggkeit ein willkommenes Kennzeichen sein, um zu entscheiden, ob solche Koks einer hohen Hitze ansgesctzt waren oder nicht, denn was oben von dem Durchglühen der Steinliohlen vor dem Lüthrohre gesagt wurte, git natürlich auch von den Aulhraciten; alle werden wach einer solchen Erhitzung gut leitend und auch bei deuen, welche für sicu schon leitend sind, zeight sich diese Eigenschaft nach solchem Durchgühen erhōht. Dass dieses Guühen übrigens gar kein ausserordentlicher llitzgrad ist, kann man daraus entnehmen, dass es noch lange nicht hinreicht, um $z$. B. teichllüssige Silicate, die nicht viel schwerer schmelzbar sind als gewöhnliches Calas, in Stüclien von halber Erissengrósse in vollkommenen Fluss zu Juringen.

ob durch fortsclireitende Zersetzung ron Steinkollen ohne Feuer ein leitender Anthracit entstehen künıe, wissen wir nicht, der Anthracit von Hol scheint darür zu sprechen. Ein durch Verkoling entstandener leitender Anthracit mag aber in sehr verschiedenen hohen Hitzgraden sich befunden habeu, hierüber grebt unser Experinent lieinen Aufschluss; zeigt dagegen mit aller Bestimmtheit, dass ein nichlleirender Anthracit in keine' heftigen Glihlhitze sich befiunden habe, in keinem Falle in einer Hitze, wie sie erforderlich wäre, um Sandsteine zu fritten oder die beobachteten durchsetzenden Gesteine zum Schmelzen zu bringen.

Die geeigneten Untersuchungen der hier einschlägigen Fälle dürften uns wichtige Aufschlüsse über manche Gesteinsbildungen und geologische Erscheinungen geben, wie sie kaum auf irgend einen andern Wege so leicht und sicher erhalten werden können. 